\title{
Relação entre aptidão aeróbia e capacidade de sprints repetidos no futebol: efeito do protocolo
}

\author{
Relationship between aerobic fitness and repeated sprint \\ ability in soccer: protocol effect
}

\author{
Juliano Fernandes da Silva \\ Luiz Guilherme Antonacci Guglielmo \\ Naiandra Dittrich \\ Leandro Teixeira Floriano ${ }^{1}$ \\ Francimara Budal Arins
}

1 Universidade Federal de Santa Catarina, Centro de Desportos. Laboratório de Esforço Físico. Florianópolis, SC, Brasil.

Recebido em 14/06/10 Revisado em 15/09/10 Aprovado em 21/10/10
Resumo - O objetivo do estudo foi investigar a associação entre variáveis fisiológicas relacionadas à aptidão aeróbia determinadas de forma contínua em corrida na esteira (pico de velocidade da esteira: $\mathrm{PV}_{\mathrm{EST}}$, consumo máximo de oxigênio: $\mathrm{VO}_{2}$ max, velocidade correspondente ao $\mathrm{VO}_{2}$ max: $\mathrm{vVO}_{2}$ max e velocidade referente ao limiar anaeróbio: vLAn) e intermitente com mudança de sentido (pico de velocidade: PV) com a capacidade de sprints repetidos (CSR) em jogadores de futebol. Vinte e nove atletas (17,9 $\pm 1,0$ anos; 178,7 $\pm 5,2 \mathrm{~cm} ; 73,6 \pm 6,7 \mathrm{~kg} ; 11,1 \pm 1,3 \%$ gordura) realizaram, em dias diferentes, os seguintes testes: 1) protocolo incremental na esteira rolante para determinar o $\mathrm{PV}_{\mathrm{EST}}$, $\mathrm{O} \mathrm{VO}_{2} \mathrm{max}$, a $\mathrm{vVO}_{2}$ max e a vLAn; 2) teste incremental de corrida intermitente (TCar) para determinar o PV e 3) teste de Bangsbo para avaliar a CSR e determinar o tempo médio (TM), melhor tempo (MT) e índice de fadiga (IF). Foi realizada a correlação linear de Pearson, adotando-se o valor de p $<0,05$ para significância. O PV obtido no TCar apresentou maior correlação com o TM e o MT ( $\mathrm{r}=-0,70, \mathrm{p}<0,01 ; \mathrm{r}=-0,51, \mathrm{p}<0,01$, respectivamente) do teste de CSR, que a $\mathrm{VVO}_{2} \max (\mathrm{r}=-0,43, \mathrm{p}<0,05 ; \mathrm{r}=-0,14 \mathrm{p}>0,05)$ e o $\mathrm{PV}_{\mathrm{EST}}(\mathrm{r}=-0,42, \mathrm{p}<0,05 ; \mathrm{r}=-0,14$, $\mathrm{p}>0,05)$ determinados no modelo de corrida em linha reta na esteira. Pode-se concluir que a relação entre aptidão aeróbia e CSR é dependente do tipo de protocolo utilizado (contínuo em linha reta vs intermitente com mudança de sentido) e da variável aeróbia utilizada (capacidade vs potência).

Palavras-chave: Aptidão aeróbia; Capacidade de sprints repetidos; Futebol; Variáveis fisiológicas.

Abstract - The aim of this study was to evaluate the association between physiological variables related to physical fitness determined by continuous straight running on a treadmill (peak velocity on treadmill: $P V_{T R E}$, maximum oxygen uptake: $\mathrm{VO}_{2} \max$, minimum velocity needed to reach $\mathrm{VO}_{2} \max : \mathrm{VO}_{2} \max$, and velocity at the anaerobic threshold: vAT) and intermittent running with directional changes (peak velocity: $P V$ ) and repeated sprint ability (RSA) in soccer players. Twenty-nine athletes $(17.9 \pm 1.0$ years, $178.7 \pm 5.2 \mathrm{~cm}, 73.6 \pm 6.7 \mathrm{~kg}$, and $11.1 \pm 1.3 \%$ body fat $)$ performed the following tests on different days: 1) incremental protocol on a treadmill to determine $P V_{T R E}, V_{2} \max , v \mathrm{VO}_{2} \max$, and $\left.v \mathrm{AT} ; 2\right)$ incremental intermittent running test to determine $P V$, and 3) Bangsbo test to evaluate RSA and to determine the mean time (MT), fastest time (FT), and fatigue index (FI). Pearson's correlation coefficient was used and a level of significance of 5\% was adopted. PV obtained in the intermittent running test showed a higher correlation with MT and FT of the RSA test $\left(r=-0.70, p<0.01 ; r=-0.51, p<0.01\right.$, respectively) than $v \mathrm{VO}_{2} \max$ $(r=-0.43, p<0.05 ; r=-0.14 p>0.05)$ and $P V_{T R E}(r=-0.42, p<0.05 ; r=-0.14, p>0.05)$ determined by straight running on a treadmill. In conclusion, the relationship between physical fitness and RSA depends on the type of protocol (continuous line running vs. intermittent with directional changes) and the aerobic index used (capacity vs. power).

Key words: Physical fitness; Repeated sprint ability; Soccer; Physiological variables. 


\section{INTRODUÇÃO}

O futebol é considerado um esporte complexo, visto que o desempenho depende de um alto nível técnico, tático, físico e psicológico. Entretanto, apesar da importância da técnica e tática, as adaptações físicas são frequentemente enfatizadas nos treinamentos, pois tal progresso busca aprimoramentos anatômicos, funcionais, biomecânicos e fisiológicos específicas ao futebol' ${ }^{1}$. No entanto, para um melhor delineamento dos programas de treinamento é importante o conhecimento da caracterização fisiológica da modalidade ${ }^{2}$, assim como, verificar quais índices fisiológicos melhor se correlacionam com o desempenho dos atletas ${ }^{3}$.

Estudos recentes têm destacado a potência aeróbia (consumo máximo de oxigênio: $\mathrm{VO}_{2} \max$ e velocidade correspondente ao $\mathrm{VO}_{2}$ max: vVO$\left.{ }_{2} \max \right)$, a velocidade referente ao limiar anaeróbio (vLAn) e a capacidade de sprints repetidos (CSR) como componentes da aptidão física do jogador de futebol ${ }^{1,4,5}$. A importância destas variáveis foi confirmada por Rampinini et al. ${ }^{5}$, que reportaram significantes correlações entre o pico de velocidade (PV) obtido no teste de aptidão aeróbia Montreal University Track Test adaptado e a CSR, com a distância percorrida em alta intensidade (> 19,8 $\left.\mathrm{Km} \cdot \mathrm{h}^{-1}\right)$ por jogadores de futebol durante a partida. Além disso, Helgerud et al. ${ }^{4}$ observaram melhorias no desempenho de jogadores de futebol, avaliados pelo número de sprints e pelo número de envolvimentos com bola, após a implementação de um programa de treinamento de potência aeróbia de oito semanas.

Vários testes têm sido propostos com o objetivo de aumentar a especificidade das avaliações que mensuram o PV em esportes intermitentes ${ }^{6,7}$, reproduzindo o padrão motor das modalidades. Tais testes permitem avaliar um elevado número de atletas simultaneamente, aumentam a motivação dos avaliados, apresentam baixo custo e ainda são de fácil aplicação $0^{7,8}$.

Em protocolos contínuos, o PV está associado com a velocidade do consumo máximo de oxigênio ( $\left.\mathrm{vVO}_{2} \max \right)$; entretanto, é importante ressaltar que em testes intermitentes este índice fisiológico também é influenciado pela capacidade e potência anaeróbia e capacidade neuromuscular para correr em altas velocidades?.

Desta forma, a contribuição da CSR e da aptidão aeróbia para o desempenho no futebol está bem descrita na literatura ${ }^{1,4,5}$. Entretanto, ainda são conflitantes os achados acerca da relação entre aptidão aeróbia e CSR. Tomlin e Wenger ${ }^{10}$, em uma revisão sobre CSR e aptidão aeróbia, reportaram que existe uma associação entre as variáveis; contudo, os autores também afirmaram que esta relação pode não ser causa efeito. Aziz et al. ${ }^{11}$ reportaram uma correlação moderada $(r=-0,346, p<0,05)$ entre o $\mathrm{VO}_{2}$ max e CSR (tempo total dos sprints) (8 x 40-m sprints com $30 \mathrm{~s}$ de recuperação entre os sprints). Porém, utilizando um diferente teste de CSR (6 x 20-m sprints com $20 \mathrm{~s}$ de recuperação entre os sprints), Aziz et al. ${ }^{12}$ não encontraram relação entre $\mathrm{o} \mathrm{VO}_{2}$ max e o desempenho no teste de CSR utilizado. É importante enfatizar que a idade e o nível de treinamento dos atletas nos dois estudos eram diferentes, além de ambos os estudos utilizarem testes incrementais com características continuas, fatores estes que podem ter influenciado os resultados da comparação.

Tendo em vista que exercícios com mudança de sentido exigem uma frenagem, seguida de uma aceleração, verifica-se que a força e a resistência muscular dos membros inferiores também são passíveis de serem aumentadas neste modelo de esforço. Assim, em comparação com protocolos que utilizam corrida em linha reta, os exercícios com mudança de sentido poderiam apresentar uma demanda aeróbia maior dos membros inferiores ${ }^{13,14}$. Desta forma, parece existir uma lacuna na literatura sobre a relação de indicadores anaeróbios com variáveis aeróbias determinados em diferentes modelos de corrida (contínua em linha reta vs intermitente com mudança de sentido).Tais achados poderão contribuir para o delineamento dos modelos de treino que visam combinar treinamento aeróbio e CSR para atletas de futebol.

Assim, o objetivo do presente estudo foi investigar a associação entre variáveis fisiológicas relacionadas à aptidão aeróbia determinadas de forma contínua em corrida na esteira $\left(\mathrm{PV}, \mathrm{vVO}_{2} \max\right.$, $\mathrm{VO}_{2} \max$ ) e intermitente com mudança de sentido (PV) com a CSR (tempo médio, melhor tempo, índice de fadiga) em jogadores de futebol de elite.

A hipótese do presente estudo é que o PV determinado no teste incremental de corrida intermitente (TCar) apresenta maior correlação com a CSR que os índices determinados no modelo sem mudança de sentido ( $\mathrm{VVO}_{2} \max , \mathrm{VO}_{2} \max$ e $\mathrm{PV}$ ), visto que as corridas com mudança de sentido apresentam respostas fisiológicas e neuromusculares que, em tese, assemelham-se mais às corridas de protocolos de CSR que as corridas contínuas em linha reta. 


\section{PROCEDIMENTOS METODOLÓGICOS}

\section{Sujeitos}

Vinte e nove jogadores de futebol treinados $(17,9 \pm$ 1,0 anos; $178,7 \pm 5,2 \mathrm{~cm} ; 73,6 \pm 6,7 \mathrm{~kg} ; 11,1 \pm 1,3$ $\%$ gordura) de duas equipes de nível nacional (A, B) participaram do estudo. A equipe $A(n=15)$ foi campeã da categoria (taça júnior) dois meses antes do estudo, enquanto que a equipe $B(n=14)$ também estava entre as melhores do país na categoria. $\mathrm{O}$ termo de consentimento informado foi assinado por todos os atletas, após uma breve explicação detalhada sobre os principais objetivos, benefícios e riscos que envolviam esta investigação. Os participantes foram informados de que estavam livres para se retirar do estudo a qualquer momento sem penalidade. Todos os procedimentos foram aprovados pelo Comitê de Ética da Universidade Federal de Santa Catarina (protocolo-384/07).

\section{Capacidade de Sprints Repetidos (CSR)}

Previamente ao teste de CSR, cada atleta realizou um período de quinze minutos de alongamento e aquecimento, seguido por cinco minutos de repouso. O teste de CSR envolveu sete sprints máximos de $34,2 \mathrm{~m}^{15}$. Em cada sprint foram realizadas três mudanças de sentido e um período de recuperação de 25 segundos, enquanto o atleta se posicionava para um novo sprint. O tempo de cada sprint foi mensurado por meio do sistema de fotocélulas (CEFISE® - Speed Test 6.0).

As seguintes variáveis foram derivadas do teste de CSR:

- Melhor tempo (MT): O melhor tempo do atleta nos sete sprints;

- Tempo médio (TM): A média de tempo dos sete sprints;

- Índice de fadiga (IF):

$$
\mathrm{IF}=\left\{\begin{array}{ll}
\frac{\text { 7tempos }}{M T^{*} 7} & -1 \times 100
\end{array}\right\}
$$

\section{Teste de laboratório}

Para a determinação do $\mathrm{VO}_{2} \max , \mathrm{IVO}_{2} \max$, vLAn, e PV, foi realizado um protoloco incremental em esteira ergométrica (Imbramed Millenium Super ATL, 10.200, Brazil). A velocidade inicial foi $9,0 \mathrm{~km} \cdot \mathrm{h}^{-1}$ (1\% inclinação) com incrementos de $1,2 \mathrm{~km} \cdot \mathrm{h}^{-1}$ a cada 3 minutos até a exaustão voluntária, intercalados com intervalos de 30 s para dosagem do lactato sanguíneo. Durante o teste, cada sujeito foi encorajado verbalmente para realizar o máximo esforço.
O consumo de oxigênio $\left(\mathrm{VO}_{2}\right)$ foi mensurado respiração a respiração, utilizando-se um analisador de gás, que foi calibrado de acordo com as recomendações do fabricante antes de cada teste $\left(\mathrm{K} \mathrm{b}^{2}\right.$, Cosmed, Rome, Italy). Os dados foram reduzidos às médias de 15 segundos e o $\mathrm{VO}_{2}$ max foi considerado como sendo o maior valor nestes intervalos de 15 s. $\mathrm{O} \mathrm{VO}_{2} \max$ foi definido pelos critérios propostos por Taylor et al. ${ }^{16} \mathrm{e} \mathrm{Lacour} \mathrm{et} \mathrm{al.}{ }^{6} \mathrm{~A}_{\mathrm{vVO}} \max$ foi considerada como sendo a menor intensidade de exercício na qual ocorreu o $\mathrm{VO}_{2} \max$. A vLAn foi determinada como a intensidade correspondente à concentração fixa de $3,5 \mathrm{mmol} . \mathrm{L}^{-1}$, a qual apresenta correlação $(r=0.80, p<0.05)$ com a máxima fase estável de lactato (MLSS) ${ }^{17}$.

\section{Teste de Carminatti (Tcar)}

Os atletas foram submetidos a um teste incremental máximo, com multi estágios de 90 segundos de duração, em sistema "ida-e-volta", constituído de 5 repetições de 12 segundos de corrida (distância variável), intercaladas por 6 segundos de caminhada ( \pm 5 metros). O ritmo foi ditado por um sinal sonoro (bip), em intervalos regulares de 6 segundos, que determinam a velocidade de corrida a ser desenvolvida nos deslocamentos entre as linhas paralelas demarcadas no solo e também sinalizadas por cones.

A velocidade inicial foi de $9,0 \mathrm{~km} \cdot \mathrm{h}^{-1}(15 \mathrm{~m}$ distância inicial), com incrementos de $0,6 \mathrm{~km} \cdot \mathrm{h}^{-1}$ a cada estágio, até a exaustão voluntária, mediante aumentos sucessivos de $1 \mathrm{~m}$ a partir da distância inicial. Quando o atleta foi incapaz de completar o último estágio, a correção do pico de velocidade $\left(\mathrm{PV}_{\mathrm{TCAR}}\right)$ foi baseada na equação de Kuipers et al. ${ }^{18}$ : PV $\left(\mathrm{km} \cdot \mathrm{h}^{-1}\right)=\mathrm{v}+[(\mathrm{nv} / 10) * 0.6]$, onde "v" é a velocidade de corrida do último estágio completado, "nv" é o número de voltas no estágio incompleto, "10" é o total de número de voltas (correndo) no estágio e "0,6" é o incremento de velocidade (adaptado Kuipers et al.) ${ }^{18}$. Quando os sujeitos não alcançavam a linha final duas vezes consecutivas o teste era encerrado.

\section{Análise Estatística}

O programa Statistical Package for Social Sciences (SPSS 13.5 for Windows) foi utilizado para realização da análise estatística. Foi empregada a análise descritiva (média e desvio-padrão) para apresentação dos valores referentes aos testes realizados, seguido do teste de Shapiro-Wilk $(\mathrm{n}<50)$ para verificar a normalidade dos dados. A escala de magnitudes proposta por Hopkins ${ }^{19}$ foi usada para interpretar os coeficientes de correlação: $<0,1=$ trivial; 0,1-0,29 
= pequena; $0,30-0,49=$ moderada; $0,50-0,69=$ alta; $0,70-0,90=$ muito alta; $>0,90=$ quase perfeita. Foi utilizada correlação linear de Pearson para determinar a relação entre as variáveis aeróbias (PV, $\mathrm{PV}_{\mathrm{EST},}, \mathrm{VO}_{2} \max , \mathrm{IVO}_{2} \max , \mathrm{vLAn}$ ) com as variáveis do teste de CSR (MT, TM, IF). O nível de significância adotado foi de $\mathrm{p}<0,05$.

\section{RESULTADOS}

Os dados descritivos do teste TCar, do protocolo laboratorial em esteira rolante e do teste de CSR estão apresentados na tabela 1 .

Tabela 1. Valores descritivos de variáveis aeróbias e anaeróbias.

\begin{tabular}{lc}
\hline Variável $(\mathrm{n}=29)$ & Média $\pm \mathrm{DP}$ \\
\hline Aptidão aeróbia & \\
$\mathrm{VO}_{2} \max \left(\mathrm{mL} \cdot \mathrm{kg}^{-1} \cdot \mathrm{min}^{-1}\right)$ & $63,2 \pm 4,9$ \\
$\mathrm{VVO}_{2} \max \left(\mathrm{km} \cdot \mathrm{h}^{-1}\right)$ & $16,8 \pm 1,0$ \\
$\mathrm{VLAn}\left(\mathrm{km} \cdot \mathrm{h}^{-1}\right)$ & $13,5 \pm 1,2$ \\
$\mathrm{PV}$ & $17,2 \pm 0,8$ \\
$\mathrm{PV} \mathrm{V}_{\mathrm{TCAR}}\left(\mathrm{km} \cdot \mathrm{h}^{-1}\right)$ & $16,5 \pm 0,9$ \\
$\mathrm{Capacidade} \mathrm{de} \mathrm{sprints} \mathrm{repetidos}(\mathrm{CRS})$ & \\
$\mathrm{TM}(\mathrm{s})$ & $6,56 \pm 0,23$ \\
$\mathrm{MT}(\mathrm{s})$ & $6,30 \pm 0,24$ \\
$\mathrm{IF}(\%)$ & $4,0 \pm 1,9$ \\
\hline
\end{tabular}

$\mathrm{VO}_{2} \max =$ consumo máximo de oxigênio; $\mathrm{vVO}_{2} \max =$ velocidade relacionada ao $\mathrm{VO}_{2} \max ; \mathrm{vLAn}=$ velocidade referente à concentração de 3,5 mmol. $\mathrm{L}^{-1} ; \mathrm{PV}_{\mathrm{TCAR}}=$ pico de velocidade atingido no TCar; $\mathrm{PV}_{\mathrm{EST}}=$ pico de velocidade atingido na esteira; $M T=$ melhor tempo obtido no teste de CSR; $\mathrm{TM}=$ tempo médio obtido no teste de CSR; IF = Índice de fadiga obtido no teste de CSR.

Os coeficientes de correlação (r) entre a vLAn e os índices determinados no teste de CSR (TM, IF) estão descritos nas figuras 1 e 2 .

$\mathrm{Na}$ tabela 3, estão apresentadas as associações entre as variáveis referentes à potência aeróbia e os índices do teste de CSR.

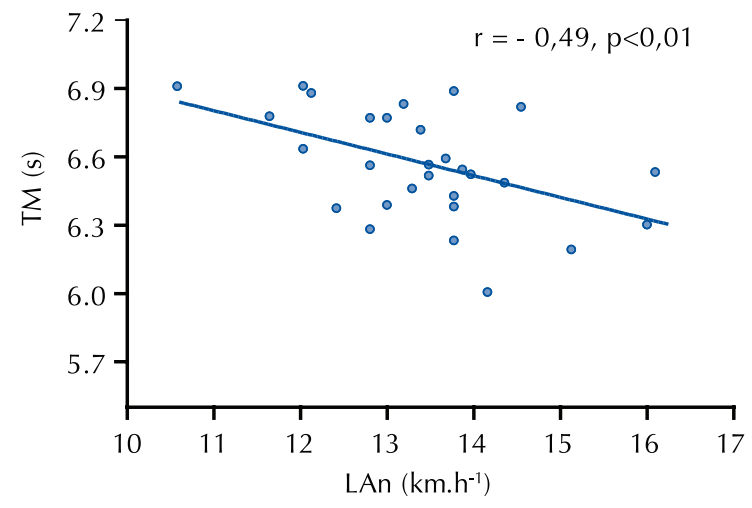

Figura 1. Correlação entre tempo médio (TM) e velocidade referente ao limiar aeróbio (vLAn).

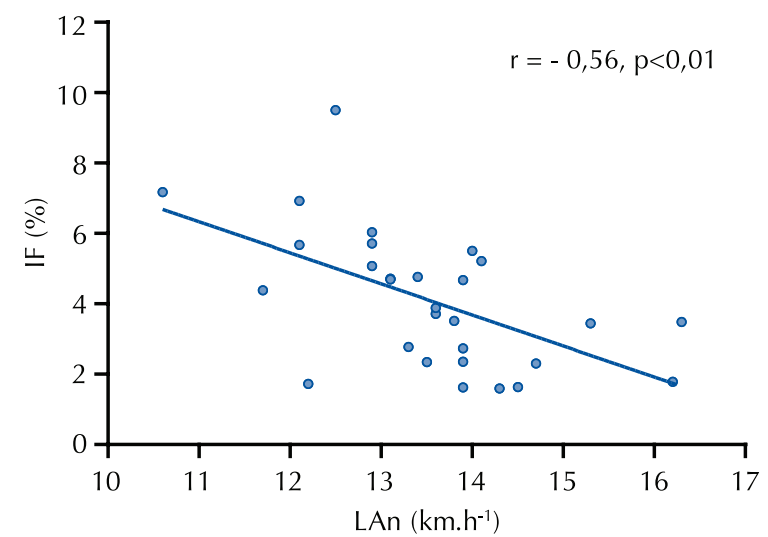

Figura 2. Correlação entre índice e fadiga (IF) e velocidade referente ao limiar aeróbio (vLAn).

Tabela 3. Coeficientes de correlação (r) entre os índices de potência aeróbia e CSR.

\begin{tabular}{lccc}
\hline Variáveis $(\mathrm{n}=29)$ & $\begin{array}{c}\mathrm{MT} \\
(\mathrm{s})\end{array}$ & $\begin{array}{c}\mathrm{TM} \\
(\mathrm{s})\end{array}$ & $\begin{array}{c}\mathrm{IF} \\
(\%)\end{array}$ \\
\hline $\mathrm{VO}_{2} \max \left(\mathrm{ml} \cdot \mathrm{kg}^{-1} \cdot \mathrm{min}^{-1}\right)$ & 0,12 & 0,08 & $-0,39 *$ \\
$\mathrm{VVO}_{2} \max \left(\mathrm{km} \cdot \mathrm{h}^{-1}\right)$ & $-0,14$ & $-0,43^{*}$ & $-0,52^{* *}$ \\
$\mathrm{PV}_{\mathrm{EST}}\left(\mathrm{km} \cdot \mathrm{h}^{-1}\right)$ & $-0,14$ & $-0,42^{*}$ & $-0,52^{* *}$ \\
$\mathrm{PV}_{\mathrm{TCAR}}\left(\mathrm{km} \cdot \mathrm{h}^{-1}\right)$ & $-0,51^{* *}$ & $-0,70^{* *}$ & $-0,31$ \\
\hline
\end{tabular}

${ }^{* *} \mathrm{p}<0,01, * \mathrm{p}<0,05$.

$\mathrm{VO}_{2} \max =$ consumo máximo de oxigênio; $\mathrm{vVO}_{2} \max =$ velocidade relacionada ao $\mathrm{VO}_{2}$ max; $\mathrm{vLAn}=$ velocidade referente à concentração de 3,5 mmol.L-1 $;$ PV $\mathrm{EST}=$ pico de velocidade atingido na esteira; $\mathrm{PV}_{\mathrm{TCAR}}=$ pico de velocidade atingido no Tcar.

\section{DISCUSSÃO}

O principal achado deste estudo foi que os índices referentes à CSR (TM, MT) apresentaram maior associação com o PV determinado no modelo intermitente com mudança de sentido (TCar) $(r=-0,70$, $\mathrm{p}<0,01 ; \mathrm{r}=-0,51, \mathrm{p}<0,01$, respectivamente), que com a vVO $\max (r=-0,43, p<0,05 ; \mathrm{r}=-0,14 \mathrm{p}>0,05)$ e o $\mathrm{PV}_{\mathrm{EST}}(\mathrm{r}=-0,42, \mathrm{p}<0,05 ; \mathrm{r}=-0,14, \mathrm{p}>0,05)$ determinados no modelo de corrida em linha reta. Assim, foi possível aceitar a hipótese do nosso estudo, pois o modelo de exercício interfere nas associações entre variáveis de potência aeróbia e CSR, demonstrando que o PV, quando determinado em protocolos intermitentes com mudanças de sentido, é mais fortemente correlacionado com a CSR, que em protocolos contínuos de corrida em linha reta.

Os dados de $\mathrm{VO}_{2} \max$ dos futebolistas deste estudo estão de acordo com a literatura, que apresenta uma variação de 55 a $68 \mathrm{~mL} \cdot \mathrm{kg}^{-1} \cdot \mathrm{min}^{-1}{ }^{2}$, sendo considerado os valores acima de $60 \mathrm{~mL} \cdot \mathrm{kg}^{-1} \cdot \mathrm{min}^{-1}$ como mínimo para um elevado desempenho, em nível internacional ${ }^{20}$. Os atletas que apresentam elevado $\mathrm{VO}_{2} \max$, possuem características favoráveis para o desempenho, como aumento nos estoques de glicogênio muscular ${ }^{3}$, no grau de recuperação ${ }^{21}$, no número 
de sprints $^{22}$, na mobilização e utilização de gorduras, poupando o glicogênio para momentos decisivos das partidas ${ }^{23}$, além de percorrerem uma maior distância em altas intensidades e apresentarem elevado número de situações de envolvimento com a bola 4 .

Neste estudo, também, foi determinada a vVO${ }_{2}$ max, que reflete a combinação entre a potência aeróbia máxima e a economia de movimento. A $\mathrm{vVO}_{2}$ max é considerada um importante preditor de desempenho aeróbio e para controle dos efeitos do treinamento ${ }^{24}$. Os dados referentes à $v \mathrm{VO}_{2}$ max do presente estudo são inferiores aos achados de Santos ${ }^{25}$, porém superiores aos dados reportados por Stolen, Chamari, Castagna e Wisloff ${ }^{26}$ para jogadores de futebol. A diferença nos valores encontrados pode estar associada com a faixa etária das amostras, períodos de treinamento, mas, principalmente, aos protocolos utilizados, pois apesar de todos os estudos terem sido realizados no laboratório, o incremento, a duração do estágio e a inclinação da esteira são fatores que interferem no valor da $\mathrm{vVO}_{2} \max ^{26}$.

É importante ressaltar que a determinação do $\mathrm{VO}_{2} \max$ e da $\mathrm{vVO}_{2}$ max necessita de avaliações individualizadas, aparelhos de alto custo e pessoal especializado. Neste sentido, o PV em testes de campo é um índice de fácil obtenção, elevada validade e reprodutibilidade, e por isso vem sendo utilizado para avaliação da potência aeróbia de futebolistas. Segundo Ahmaidi et al. ${ }^{8}$, protocolos incrementais com mudança de sentido tendem a apresentar uma maior solicitação anaeróbia ao final do teste, devido ao fato de acelerar, reduzir a velocidade, parar e mudar de sentido durante o teste, causando deslocamento vertical do centro de massa e menor eficiência da passada ${ }^{8}$.

De acordo com Buchheit et al..$^{14}$, os exercícios com mudança de sentido exigem uma frenagem, seguidos por uma aceleração, assim, a importância da força e da resistência muscular dos membros inferiores também é passível de ser aumentada neste modelo de esforço. Desta forma, em comparação com a corrida em linha reta, os exercícios com mudança de sentido tendem a apresentar uma demanda aeróbia maior dos membros inferiores para uma mesma intensidade submáxima de exercício ${ }^{13}$.

Por outro lado, não foi encontrada associação entre o $\mathrm{VO}_{2} \max$ e os índices referentes a CSR. $\mathrm{O}$ fato de $\mathrm{O} \mathrm{VO}_{2} \max$ ser limitado principalmente por fatores centrais e o modelo utilizado para sua determinação ter sido contínuo em linha reta podem explicar os resultados encontrados, visto que a CSR é determinada principalmente por adap- tações periféricas ${ }^{5}$. Já a vLAn, que está associada principalmente às respostas aeróbias periféricas, apresentou correlação moderada com o TM $(\mathrm{r}=$ $0,49, \mathrm{p}<0,01)$. Desta forma, é possível considerar que tanto o tipo de variável aeróbia (capacidade ou potência; central ou periférica) como o modelo de protocolo empregado (com ou sem mudança de sentido), apresentam respostas diferentes no que diz respeito à relação entre aptidão aeróbia e CSR.

Durante exercícios de sprints repetidos, o desempenho pode ser definido como a capacidade de repetir máxima potência após prévio exercício ${ }^{27}$. A incapacidade de manter o desempenho em sprints repetidos tem sido atribuída principalmente ao acúmulo de metabólitos, tais como o aumento na concentração sanguínea de lactato $([\mathrm{La}])^{28}$, acúmulo de $\mathrm{H}^{+29}$ e depleção de fosfocreatina muscular ${ }^{30}$. Entretanto, fatores como mudanças na coordenação neuromuscular da contração muscular também tem sido relacionadas à fadiga em exercícios de sprints repetidos ${ }^{27}$.

Outro achado deste estudo foi a alta correlação entre o MT e o PV no TCar $(r=-0,51, p<0,01)$, ratificando que o PV determinado no modelo intermitente com mudança de sentido é influenciado pela capacidade do indivíduo acelerar e alcançar elevada intensidade em curtos espaços. Adicionalmente, não foi encontrada correlação entre o MT e os indicadores de potência aeróbia no protocolo contínuo de laboratório $\left(\mathrm{PV}_{\mathrm{EST}}, \mathrm{VO}_{2} \max \mathrm{e}\right.$ $\mathrm{vVO}_{2}$ max). Este fato suporta que as características intermitentes do TCar permitem avaliar aspectos anaeróbios, devido a sua relação com o MT e o TM.

Aziz et al. ${ }^{11}$, ao estudarem a relação do $\mathrm{VO}_{2} \max$ determinado em protocolo contínuo em esteira com o MT (8 X 40 m, 30 s recuperação) em teste de CSR, não encontraram correlação significante, corroborando nossos achados. A ausência de correlação entre o $\mathrm{VO}_{2}$ max e o MT sustenta a ideia que $\mathrm{o} \mathrm{VO}_{2}$ max não tem impacto significativo sobre o desempenho em sprints curtos $(<\cong 6 s)^{11,30}$.

$\mathrm{O}$ índice de fadiga no teste de CSR apresentou correlação moderada com o $\mathrm{VO}_{2} \max (\mathrm{r}=-0,39$, $\mathrm{p}<0,01)$, e grande com a $\mathrm{vVO}_{2}$ max $(\mathrm{r}=-0,52$, $\mathrm{p}<0,01)$ e o $\mathrm{PV}_{\mathrm{EST}}(\mathrm{r}=-0,52, \mathrm{p}<0,01)$, sugerindo que a queda de desempenho em sprints repetidos em modelos de esforço como o utilizado no presente estudo é influenciada pela aptidão aeróbia ${ }^{11}$.

\section{CONCLUSÕES}

De acordo com os achados do presente estudo, conclui-se que a relação entre aptidão aeróbia e CSR é dependente do tipo de protocolo utilizado 
(contínuo em linha reta vs intermitente com mudança de sentido), assim como, da variável aeróbia utilizada (capacidade vs potência), proporcionando importantes implicações para o delineamento dos modelos de treino que visam combinar treinamento aeróbio com os de CSR.

\section{REFERÊNCIAS BIBLIOGRÁFICAS}

1. Impellizzeri FM, Rampinini E, Marcora SM. Physiological assessment of aerobic training in soccer. J Sports Sci 2005;23:583-92.

2. Wisloff U, Helgerud J, Hoff J. Strength and endurance of elite soccer players. Med Sci Sports Exerc 1998; 30:462-7.

3. Hoff J, Wislöff U, Engen LC, Helgerud J. Soccer specific aerobic endurance training. Br J Sports Med 2002;36:218-21.

4. Helgerud J, Engen LC, Wisloff U, Hoff J. Aerobic endurance training improves soccer performance. Med Sci Sports Exerc 2001;33(11):1925-31.

5. Spencer M, Bishop D, Dawson B, Goodman C. Physiological and metabolic responses of repeated-sprint activities specific to field-based team sports. Sports Med 2005;35(12): 1025-44.

6. Lacour JR, Padilla-Magunacelaya S, Chatard JC, Arsac L, Bathélémy, JC. Assessment of running velocity at maximal oxygen uptake. Eur J Applied Physiol 1991;62(2):77-82.

7. Carminatti LJ, Lima-Silva AE, De-Oliveira FR. Aptidão Aeróbia em esportes intermitentes: evidências de validade de construto e resultados em teste incremental com pausas. Rev Bras Fisiol Exerc (resumo) 2004;3(1):120.

8. Ahmaidi S, Collomp K, Caillaud C, Préfaut, C. Maximal and functional aerobic capacity as assessed by two graduated field methods in comparison to laboratory exercise testing in moderately trained subjects. Int J Sports Med 1992;13(3):243-8.

9. Fernandes da Silva J, Guglielmo LGA, Floriano LT, Arins FB, Dittrich N. Aptidão aeróbia e capacidade de sprints repetidos no futebol: comparação entre as posições. Motriz 2009;15(4):861-70.

10. Tomlin DL, Wenger HA. The relationship between aerobic fitness and recovery from high intensity intermittent exercise. Sports Med 2001;31(1):1-11.

11. Aziz AR, Chia M, Teh KC. The relationship between maximal oxygen uptake and repeated sprint performance indices in field-hockey and soccer players. J Sports Med Phys Fitness 2000;40:195-200.

12. Aziz AR, Mukherjee S, Chia M, Teh KC. Relationship between measured maximal oxygen uptake and aerobic endurance performance with running repeated sprint ability in young elite soccer players. J Sports Med Phys Fitness 2007;7:401-7.

13. Brughelli M, Cronin J, Levin G, Chaouachi A. Understanding change of direction ability in sport: a review of resistance training studies. Sports Med 2008;38:1045-63.

14. Buchheit M, Bishop D, Haydar B, Nakamura FY, Ahmaidi S. Physiological responses to shuttle repeated-sprint running. Int J Sports Med 2010;26:402-409.

15. Bangsbo J. Fitness Training for Football: A scientific approach. HO+Storm, Bagsvaerd, 1994.
16. Taylor HL, Buskirk E, Hensciiel A. Maximal oxygen intake as an objective measure of cardio-respiratory performance. J Appl Physiol 1955;8:73-80.

17. Denadai, BS, Gomide, EBG, Greco, CC. The relationship between onset of blood lactate accumulation, critical velocity, and maximal lactate steady state in soccer players. J Strength Cond Res 2005;19:364-8.

18. Kuipers H, Verstappen FTJ, Keizer HA, Geurten P, VanKranenburg G. Variability of aerobic performance in the laboratory and its physiological correlates. Int J Sports Med 1985;6(4):197-201.

19. Hopkins WG. Measures of reliability in sports medicine and science. Sports Med 2000;30(1):1-15.

20. Reilly T, Bangsbo J, Franks A. Antropometric and physiological predispositions for elite soccer. J Sports Sci 2000;18:669-83.

21. Bangsbo J, Lindqvist F. Comparison of various exercise tests with endurance performance during soccer in professional players. Int J Sports Med 1992;13:125-32.

22. Smaros G. Energy usage during a football match. Proceedings of the 1st international congress on sports medicine applied to football (Abstract). Rome: 1980, 795-801.

23. Reilly T, Thomas, V. A motion analysis of work rate in different positional roles in professional football match-play. J Hum Mov Stud 1976;2:87-97.

24. Billat V, Pinoteau J, Petit B, Renoux JC, Koralsztein P. Time to exhaustion at $100 \%$ of velocity at VO2max and modeling of the relation timelimit/velocity in elite long distance runners. Eur J Appl Physiol 1994;69:271-3.

25. Santos JAR. Estudo comparativo, fisiológico, antropométrico e motor entre futebolistas de diferente nível competitivo. Rev paul educ fis 1999;13(2):146-59.

26. Stolen T, Chamari K, Castagna C, Wisloff U. Physiology of soccer an update. Sports Med 2005;35(6):501-36.

27. Mendez-Villanueva A, Hamer P, Bishop D. Fatigue Responses during repeated sprints matched for initial mechanical output. Med Sci Sport Exerc 2007;39: 2219-25.

28. Thomas C, Sirvent P, Perrey S, Raynaud E, Mercier J. Relationships between maximal muscle oxidative capacity and blood lactate removal after supramaximal exercise and fatigue indexes in humans. J Appl Physiol 2004;97:2132-8.

29. Glaister M. Multiple sprint work: physiological responses, mechanisms of fatigue and the influence of aerobic fitness. Sports Med 2005;35:757-77.

30. Gaitanos GC, Williams C, Boobis LH, Brooks S. Human musclemetabolism during intermittent maximal exercise. J Appl Physiol 1993;75:712-9.
Endereço para correspondência
Juliano Fernandes da Silva
Universidade Federal de Santa Catarina
Centro de Desportos - Laboratório de Esforço Físico
Campus Universitário da Trindade, s/n
CEP: 88040-900 Florianópolis (SC) Brasil
E-mail: jufesi23@yahoo.com.br 